\title{
Activation of hepatic stellate cells is suppressed by microRNA-150
}

\author{
JIANJIAN ZHENG ${ }^{1 *}$, ZHUO LIN $^{2 *}$, PEIHONG DONG ${ }^{2}$, ZHONGQIU LU $^{3}$, SHENMENG GAO $^{4}$, \\ XIAOQIAN CHEN ${ }^{5}$, CUNZAO WU ${ }^{6}$ and FUJUN YU ${ }^{2}$ \\ ${ }^{1}$ Wenzhou Key Laboratory of Surgery; ${ }^{2}$ Department of Infectious Diseases; ${ }^{3}$ Emergency Department; \\ ${ }^{4}$ Laboratory of Internal Medicine; ${ }^{5}$ Laboratory of Nephrology; ${ }^{6}$ Institute of Organ Transplantation, \\ The First Affiliated Hospital of Wenzhou Medical College, Wenzhou, Zhejiang 325000, P.R. China
}

Received January 7, 2013; Accepted February 15, 2013

DOI: $10.3892 /$ ijmm.2013.1356

\begin{abstract}
RNAs (miRNAs) have recently been reported to be involved in the progression of liver fibrosis. It has previously been shown that miR-150 can inhibit the activation of hepatic stellate cells (HSCs) via the inhibition of C-myb expression. However, the reduced $\mathrm{C}$-myb expression is not responsible for all the effects of miR-150, there may be other molecular mechanisms for the suppression of HSCs by miR-150. In this study, gene array analysis was performed to analyze the miRNAs that were differentially expressed between LX- 2 cells induced by transforming growth factor- $\beta 1$ (TGF- $\beta 1$ ) and the control. Our results indicated that the expression of miR-150 was significantly reduced during liver fibrosis. Of note, the reduction of miR-150 induced by TGF- $\beta 1$ was in a dose- and time-dependent manner. In addition, miR-150 overexpression in LX-2 cells resulted in the inhibition of cell proliferation and the reduction of extracellular matrix proteins and $\alpha$-smooth muscle actin ( $\alpha$-SMA). However, there was no significant change in the rate of apoptosis in cells transfected with miR-150 mimics compared with the control. Sp1, a mediator of $\alpha-1$ (I) collagen (Col1 A1) expression, and Col4A4 were found to be the targets for miR-150. Also, miR-150 mimics were found to decrease the expression of Sp1 and Col4A4. Smad2 and p-Smad2, the upstream mediators of Sp1, were not affected by miR-150. The same result was also seen in the levels of Smad3 and p-Smad3. Collectively, we conclude that miR-150 can reduce type I and IV collagen by directly binding to $\mathrm{Sp} 1$ and Col4A4 without the involvement of upstream of the TGF- $\beta /$ Smad pathway.
\end{abstract}

\section{Introduction}

Cirrhosis, which is induced by various factors such as chronic viral hepatitis, autoimmune hepatitis, fatty liver disease, or

Correspondence to: Dr Fujun Yu, Department of Infectious Diseases, The First Affiliated Hospital of Wenzhou Medical College, 2 Fuxue Lane, Wenzhou, Zhejiang 325000, P.R. China

E-mail: tjyufujun@163.com

*Contributed equally

Key words: microRNA-150, hepatic stellate cells, liver fibrosis hereditary metabolic disorders, is a major cause of morbidity and mortality worldwide (1-4). Due to the stimulation of hepatocellular damage and inflammation, cirrhosis is characterized by the remodeling of liver tissues with excess deposition of extracellular matrix (ECM) components such as collagens. Liver fibrosis is the intermediate link in the progression from chronic liver disease to cirrhosis. Therefore, early prevention or reversion of liver fibrosis is key in improving the prognosis of various chronic liver diseases. In the progression of liver fibrosis, hepatic stellate cells (HSCs), which may be the most important cell type for the production of collagens, are considered to be responsible for the accumulation of ECM proteins (4). HSCs become activated in response to inflammatory stimuli and undergo myofibroblastic transdifferentiation. Therefore, a possible therapeutic strategy is to treat liver fibrosis by suppressing the activation of HSCs.

microRNAs (miRNAs) are a class of small, evolutionarily conserved, non-coding RNAs, which suppress the expressions of protein coding genes by base pairing with the 3' untranslated region ( $3^{\prime}$ UTR) of their target messenger RNAs (mRNAs) (5). miRNAs can repress gene expression by either of two mechanisms: target mRNAs are degraded by perfect or nearly perfect pairing, or translational repression is caused by the imperfect pairing. To date, the number of known miRNAs has grown exponentially, and more than 1,000 miRNAs are known to be encoded by the human genome (6), half of which have been experimentally validated (7). miRNAs have been associated with numerous basic cellular processes such as cell death, proliferation and differentiation (8-11). Furthermore, the dysregulation of miRNAs has also been correlated with a wide spectrum of human diseases such as cancer (12). For example, miR-17-5p is found to be overexpressed in hepatocellular carcinoma (HCC) and increased miR-17-5p expression levels are correlated with poor prognosis of patients with HCC $(13,14)$.

In addition, a number of recent reviews have shown that miRNA dysregulation is involved in the activation of HSCs. The reduced expressions of miRNA-150 and miRNA-194 are found in activated HSCs, and their overexpressions inhibit the activation and proliferation of HSCs (15). On the contrary, reduced expressions of miR-27a and -27b allow culture-activated rat HSCs to return to a quiescent phenotype with abundant vitamin A storage and decreased cell proliferation (16). Furthermore, miR-214-5p is upregulated in human 
and mouse livers in a fibrosis progression-dependent manner and the overexpression of miR-214-5p in LX-2 cells increases the expressions of fibrosis-related genes, such as matrix metalloproteinase-2 (MMP-2), MMP-9, $\alpha$-smooth muscle actin $(\alpha-\mathrm{SMA})$, and transforming growth factor- $\beta 1$ (TGF- $\beta 1)(17)$. However, little is known about the potential molecular mechanism of these miRNAs in liver fibrosis.

In the present study, we demonstrated that miR-150 was significantly downregulated in LX-2 cells treated with TGF- $\beta 1$ when compared with the control. It was confirmed that the overexpression of miR-150 inhibited the activation and proliferation of HSCs with no effect on cell survival. In addition, we found that miR-150 was a potential regulator of type IV collagen protein expression via its interaction with Col4A4 3'UTR. Our data also suggested that miR-150 may be a regulator of type I collagen expression through the Sp1 signal pathway.

\section{Materials and methods}

Cell culture. The human LX-2 cell strain was obtained from JENNIO Biological Technology, Guangdong, China. It was cultured in DMEM containing 10\% fetal bovine serum, $100 \mathrm{U} / \mathrm{ml}$ penicillin $\mathrm{G}$ sodium salt and $100 \mathrm{U} / \mathrm{ml}$ streptomycin sulfate (Gibco, Carlsbad, CA, USA). The cells were grown in a $37^{\circ} \mathrm{C}$ incubator with $5 \% \mathrm{CO}_{2}$. Exponentially growing cells were seeded in a 6 -well plate at a density of $1 \times 10^{6}$ cells/well and were then transfected with the miR-150 mimics or a negative control (Shanghai GenePharma Co., Ltd., Shanghai, China) for $24 \mathrm{~h}$. Cells were also treated with TGF- $\beta 1$ (R\&D Systems, Shanghai, China) for different experiment purposes. Cells were harvested for RNA/miRNA isolation, and whole cell extracts were subjected to western blot analysis.

Exiqon miRCURY LNA ${ }^{\mathrm{TM}}$ miRNA array. Total RNA of HSCs was extracted and labeled with a miRCURY Hy3/Hy5 labeling kit (Exiqon, Vedbaek, Denmark). Then the labeled samples were hybridized to the miRCURY LNA array version 8.0 (Exiqon). The hybridization was performed following the miRCURY LNA array manual. Following hybridization, the slides were washed by Wash buffer kit (Exiqon), dried and scanned on a GenePix 4000B array scanner (Molecular Devices Co., Sunnyvale, CA, USA). The results were carried out using unsupervised hierarchical clustering (Cluster 3.0) and TreeView analysis (Stanford University, Stanford, CA, USA).

Quantitative real-time PCR. Total RNA was extracted from LX-2 cells using the miRNeasy Mini Kit (Qiagen, Valencia, CA, USA). Also, fifty nanograms of total RNA were reversetranscribed to cDNA using the ReverTra Ace ${ }^{\circledR}$ qPCR RT Kit (Toyobo, Osaka, Japan) in accordance with the manufacturer's instructions. Gene expression was measured by real-time PCR using cDNA, SYBR-Green real-time PCR Master Mix (Toyobo), and a set of gene-specific oligonucleotide primers; $\alpha-1$ (I) collagen (Col1A1), forward, 5'-CCCGGGTTTCAGAG ACAACTTC-3' and reverse, 5'-TCCACATGCTTTATTCCAG CAATC-3'; $\alpha-2$ (I) collagen (Col1A2), forward, 5'-AAGGGT CCCTCTGGAGAACC-3' and reverse, 5'-TCTAGAGCCAGG GAGACCCA-3'; $\alpha-4$ (IV) collagen (Col4A4), forward, 5'-TGA
AGGGAAATCCCGGTGTG-3' and reverse, 5'-CAGGTGGC TCTACCAACAGG-3'; $\alpha$-SMA, forward, 5'-TCGGATGAG CTACAGAGGCACAA-3', and reverse, 5'-GTCACTCCTCAT GAAGCGCTTAGG-3'; Sp1, forward, 5'-TCGGATGAGCTAC AGAGGCACAA-3' and reverse, 5'-GTCACTCCTCATGAAG CGCTTAGG-3'; GAPDH, forward, 5'-GCACCGTCAAGGCT GAGAAC-3', and reverse, 5'-TGGTGAAGACGCCAGTG GA-3'; U6, forward, 5'-CTCGCTTCGGCAGCACA-3' and reverse, 5'-AACGCTTCACGAATTTGCGT-3'. To detect miR-150 expression, the RT reaction was performed using the TaqMan microRNA Assay (Applied Biosystems, Foster City, CA, USA) according to the manufacturer's instructions. The GAPDH and U6 snRNA (Applied Biosystems) levels were measured and used to normalize the relative abundance of mRNAs and miRNAs, respectively. The expression level $\left(2^{-\Delta \Delta C t}\right)$ of miR-150 was calculated as previously described (18).

Protein extraction and western blot assay. Proteins were subjected to sodium dodecyl sulfate-polyacrylamide gel electrophoresis and then transferred onto Immobilon-P membranes. After blocking, the membranes were incubated with primary antibodies [mouse monoclonal antibody against $\alpha$-SMA (ab5694) and rabbit polyclonal antibody against type IV collagen (ab6586); rabbit polyclonal antibody against type I collagen (ab34710) and GAPDH (ab9485); mouse monoclonal antibody against Smad2 (ab119907) and rabbit polyclonal antibody against phospho-Smad2 (p-Smad2, ab53100); rabbit polyclonal antibody against Smad3 (ab40854) and p-Smad3 (ab52903); mouse monoclonal antibody against Sp1 (ab77441) and goat polyclonal antibody against C-myb (ab62824) (Abcam, Cambridge, MA, USA)] followed by peroxidase-conjugated secondary antibodies (Fuzhou Maixin Biological Technology Co., Ltd., Fujian, China). The antigen-antibody complex was developed by enhanced chemiluminescence, exposed in the dark room and analyzed for integral absorbance (IA) of the protein bands using quantitative software, Quantity One 4.4.

Proliferation and apoptosis assays. Cells were seeded in a 96-well plate at a density of $1 \times 10^{3}$ cells/well and the cells were then transfected with the miR-150 mimics or a negative control for $24 \mathrm{~h}$ before the assessment of the proliferation and apoptosis. Cell proliferation was determined by MTT assay according to the instructions of an MTT cell proliferation assay kit (Beyotime Institute of Biotechnology). The optical density (OD) was measured at $570 \mathrm{~nm}$ on a 550 Microplate Reader (Bio-Rad, USA). The DNA fragmentation for cell apoptosis was measured using a DNA/histone-complex ELISA kit (Roche) as described (19). The OD at $490 \mathrm{~nm}$ was measured.

Luciferase activity assay. According to the targetscan analysis, oligonucleotides, which contained human Col4A4 and Sp1 3'UTR target sequence, were annealed and cloned into the pMIR-Report ${ }^{\mathrm{TM}}$ Luciferase plasmid (Applied Biosystems) following the manufacturer's protocol to generate pMIR-150 vectors including pMIR-Col4A4-150 and pMIR-Sp1-150. Col4A4-3'UTR for miR-150 (position of 1092-1099) forward, 5'-GTGCGTGCTAATGGGACTGA-3' and reverse, 5'-GGAT GCTCCTGTAACAGCCA-3'. Sp1-3'UTR for miR-150 (position of 1479-1486) forward, 5'-TGATGTGTGGGCTTCTGA 
A

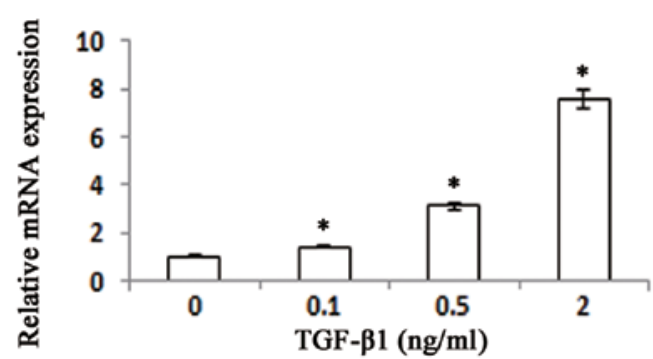

B
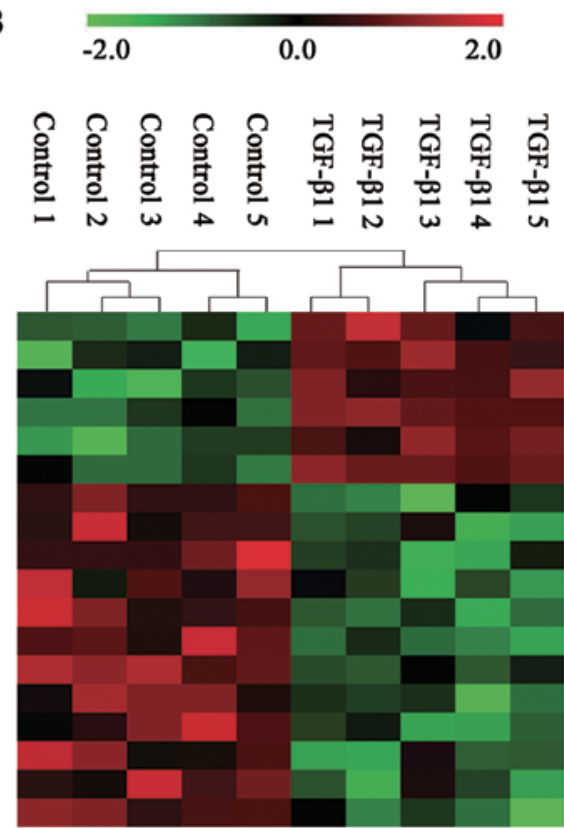

hsa-miR-34b hsa-miR-34a hsa-miR-93 hsa-miR-199-3p hsa-miR-199-5p hsa-miR-221 hsa-miR-148a hsa-miR-29a hsa-miR-194 hsa-miR-27b hsa-miR-27a hsa-miR-29c hsa-miR-150 hsa-miR-122 hsa-miR-187 hsa-miR-335 hsa-miR-677 hsa-miR-29b

Figure 1 . The deregulated mRNA induced by TGF- $\beta 1$ in LX- 2 cells. (A) The Col1A1 was dose-dependently induced by TGF- $\beta 1$. The LX-2 cells were treated with TGF- $\beta 1(0,0.1,0.5$ and $2 \mathrm{ng} / \mathrm{ml})$ for $24 \mathrm{~h}$. Bars represent mean and standard error of three independent experiments. ${ }^{*} \mathrm{P}<0.05$ compared with the control. (B) Microarray analysis for miRNAs was performed with RNA extracts from the cells treated with TGF- $\beta 1(2 \mathrm{ng} / \mathrm{ml})$ and the control Hierarchical cluster analysis of the significantly regulated miRNAs: bright green, underexpression; black, no change; bright red, overexpression.

GT-3' and reverse, 5'-ATGCTTTTATGGCTGGGCCT-3'. Sp1-3'UTR for miR-150 (position of 3953-3960) forward, 5'-AAGGTCGCAGCAGTAGCTTT-3' and reverse, 5'-GAGA CAAGGAAGACTGGGGC-3'. Empty vector pMIR without the inserts was used as a negative control. pMIR-Report $\beta$-gal control plasmid was used for transfection normalization. LX-2 cells were cultured in 24-well plates and transfected with $800 \mathrm{ng}$ of pMIR-150 or pMIR together with $100 \mathrm{ng}$ of pMIR$\beta$-gal and 20 pmol of miR-150 precursor or miRNA negative control (miR-NC) (Shanghai GenePharma Co., Ltd., Shanghai, China). Lipofectamine 2000 (Invitrogen, Carlsbad, CA, USA) was used for transfection. Forty-eight hours after transfection, luciferase and $\beta$-gal activity were measured using the DualLight System (Applied Biosystems).

Statistical analysis. Data from at least three independent experiments were expressed as the means \pm SD. Statistical analysis was performed using Student's t-test and $\mathrm{P}<0.05$ was considered to indicate a statistically significant difference.
Table I. $\log _{2}$ (TGF- $\beta 1 /$ control) ratio of differentially expressed miRNAs that reached statistical significance by t-test $(\mathrm{P}<0.01)$ and further confirmed by SAM test $(\mathrm{q}<5 \%)$.

\begin{tabular}{cc}
\hline miRNA & $\begin{array}{c}\log _{2}(\mathrm{TGF}-\beta 1 / \text { control }) \\
\text { ratio }\end{array}$ \\
\hline
\end{tabular}

Upregulated miRNAs $(\mathrm{n}=6)$

$\begin{array}{lrr}\text { miR-221 } & 1.61 & 0.0007 \\ \text { miR-199a-3p } & 1.53 & 0.0063 \\ \text { miR-34a } & 1.21 & 0.0024 \\ \text { miR-199a-5p } & 0.82 & 0.0029 \\ \text { miR-34b } & 0.63 & 0.0012 \\ \text { miR-93 } & 0.54 & 0.0041 \\ \text { Downregulated miRNAs }(\mathrm{n}=12) & \\ \text { miR-150 } & -1.86 & 0.0001 \\ \text { miR-29b } & -1.56 & 0.0017 \\ \text { miR-148a } & -1.32 & 0.0028 \\ \text { miR-122 } & -1.15 & 0.0005 \\ \text { miR-29c } & -0.97 & 0.0029 \\ \text { miR-677 } & -0.75 & 0.0077 \\ \text { miR-29a } & -0.74 & 0.0089 \\ \text { miR-187 } & -0.58 & 0.0068 \\ \text { miR-335 } & -0.56 & 0.0066 \\ \text { miR-194 } & -0.48 & 0.0021 \\ \text { miR-27a } & -0.48 & 0.0003 \\ \text { miR-27b } & -0.48 & 0.0040\end{array}$

TGF- $\beta 1$, transforming growth factor- $\beta 1$; miRNA, microRNA.

\section{Results}

TGF- $\beta 1$ causes differential expression of miRNAs in cultured $L X-2$ cells. TGF- $\beta 1$ is considered to be the most important mediator to induce HSC transformation into myofibroblasts and upregulate expression of collagen proteins (20). The expression of Col1A1 mRNA was increased dose-dependently by TGF- $\beta 1$ in cultured LX-2 cells (Fig. 1A). To screen differentially expressed miRNAs induced by TGF- $\beta 1$, we performed microarray analysis on RNA extracts from LX-2 cells with TGF- $\beta 1$ and the control. miRNAs were considered differentially expressed according to the following standards: the differences of miRNA expression levels showed significance both in unpaired Student's t-test $(\mathrm{P}<0.01)$ and significance analysis of microarray test (q-value $<5 \%$ ). The expression of several miRNAs was noted to be altered between cultured LX-2 cells with TGF- $\beta 1$ and the control (Fig. $1 \mathrm{~B}$ and Table I). miRNA-150 was among the most significantly altered miRNAs and its expression level was confirmed by quantitative real-time PCR (Fig. 2). Additionally, although there are a few studies on the involvement of miR-150 in liver fibrosis, the role of miR-150 in liver fibrosis remains unclear. Hence, we regarded miR-150 as the object of subsequent experiments.

Dose- and time-dependent reduction of miR-150 by TGF- $\beta 1$. There was a significant decrease in miR-150 expression level 
A

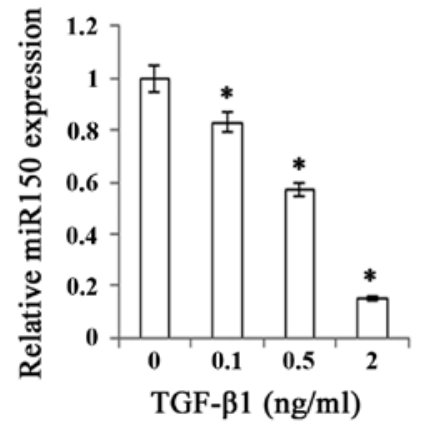

B

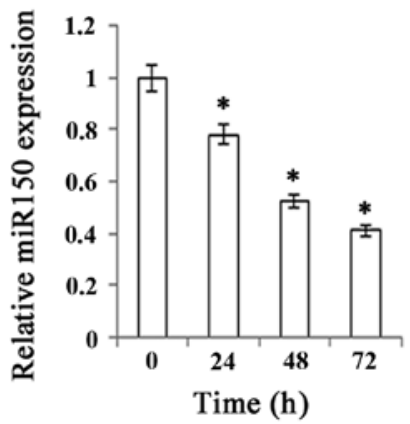

Figure 2. The effect of TGF- $\beta 1$ on miR-150 expression in LX-2 cells. (A) The downregulation of miR-150 expression was dose-dependently induced by TGF- $\beta 1$. The LX-2 cells were treated with TGF- $\beta 1(0,0.1,0.5$ and $2 \mathrm{ng} / \mathrm{ml})$ for $24 \mathrm{~h}$. (B) The downregulation of miR- 150 expression was time-dependently induced by TGF- $\beta 1$. The LX-2 cells were treated with TGF- $\beta 1(0.1 \mathrm{ng} / \mathrm{ml})$ for 0, 24, 48 and $72 \mathrm{~h}$. miR-150 expression was analyzed by real-time PCR. Bars represent mean and standard error of three independent experiments. ${ }^{*} \mathrm{P}<0.05$ compared with the control.

A

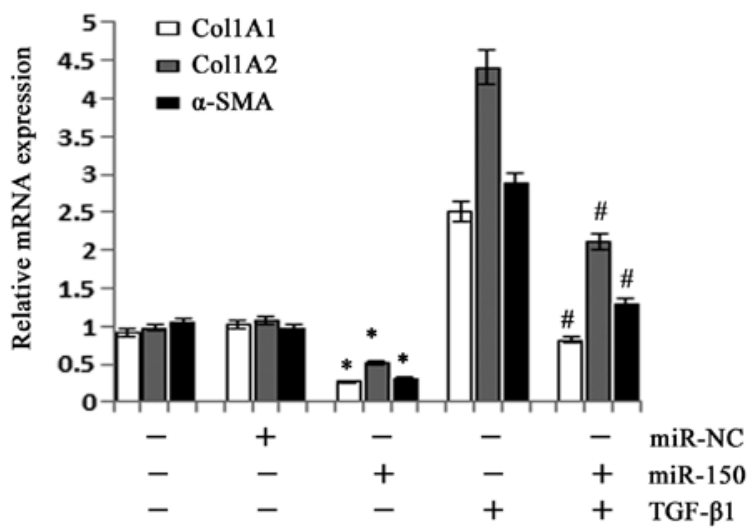

C

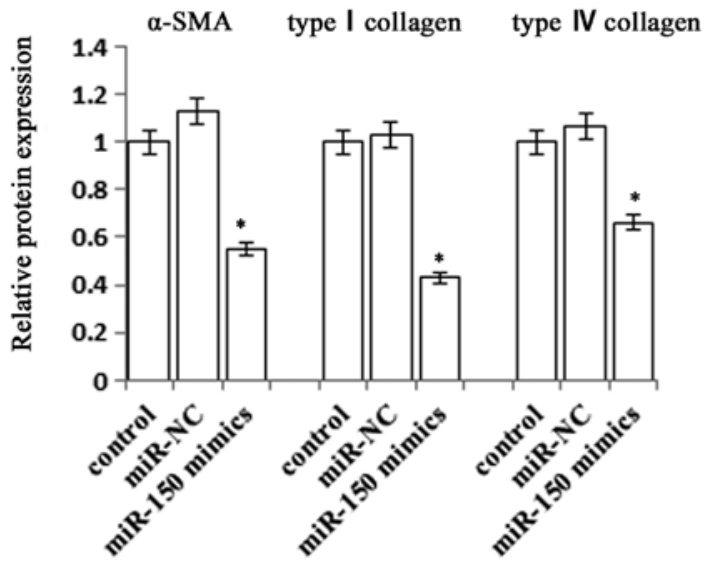

B
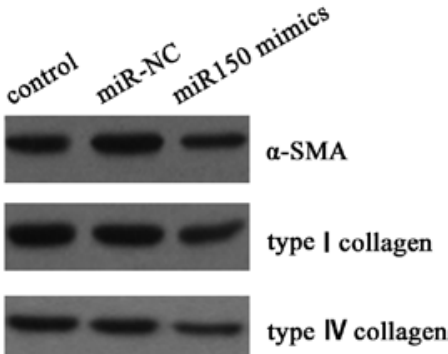

$\mathrm{D}$

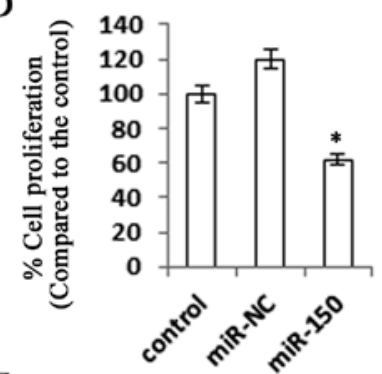

$\mathrm{E}$

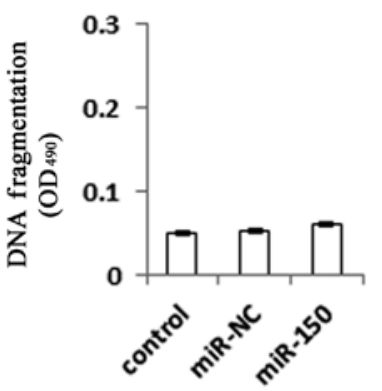

Figure 3. The effect of miR-150 overexpression on the activation of LX-2 cells. (A) The mRNA expression levels of Col1A1, Col1A2 and $\alpha$-SMA were analyzed by real-time PCR. Bars represent mean and standard error of three independent experiments. " $\mathrm{P}<0.05$ compared with the control. ${ }^{\#} \mathrm{P}<0.05$ compared with the TGF- $\beta 1$ group. (B) Protein expression levels of types I, IV collagen and $\alpha$-SMA were analyzed by western blotting. GAPDH served as an internal control. (C) Statistical analysis of type I, IV collagen and $\alpha$-SMA protein expressions. Bars represent mean and standard error of three independent experiments. "P $<0.05$ compared with the control. (D) Cell proliferation was determined by MTT assay. Bars represent mean and standard error of three independent experiments. The OD at $570 \mathrm{~nm}$ was measured. ${ }^{\mathrm{P}}<0.05$ compared with the control. (E) The apoptosis of LX-2 cells was determined by a DNA fragmentation ELISA assay. Data from one experiment repeated three times. The OD at $490 \mathrm{~nm}$ was measured.

as we increased the concentrations of TGF- $\beta 1$ from 0.1 to $2 \mathrm{ng} / \mathrm{ml}$, indicating that the inhibitory effect of TGF- $\beta 1$ on miR-150 was dose-dependent (Fig. 2A). Furthermore, miR-150 expression level was also examined at $0,24,48$ and $72 \mathrm{~h}$ after the treatment of TGF- $\beta 1$ (Fig. 2B). It was significantly decreased with the prolongation of time, the lowest being at 
A

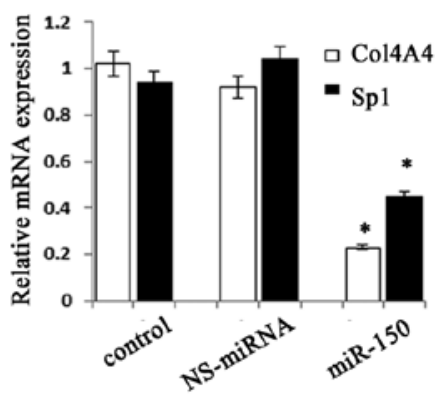

C

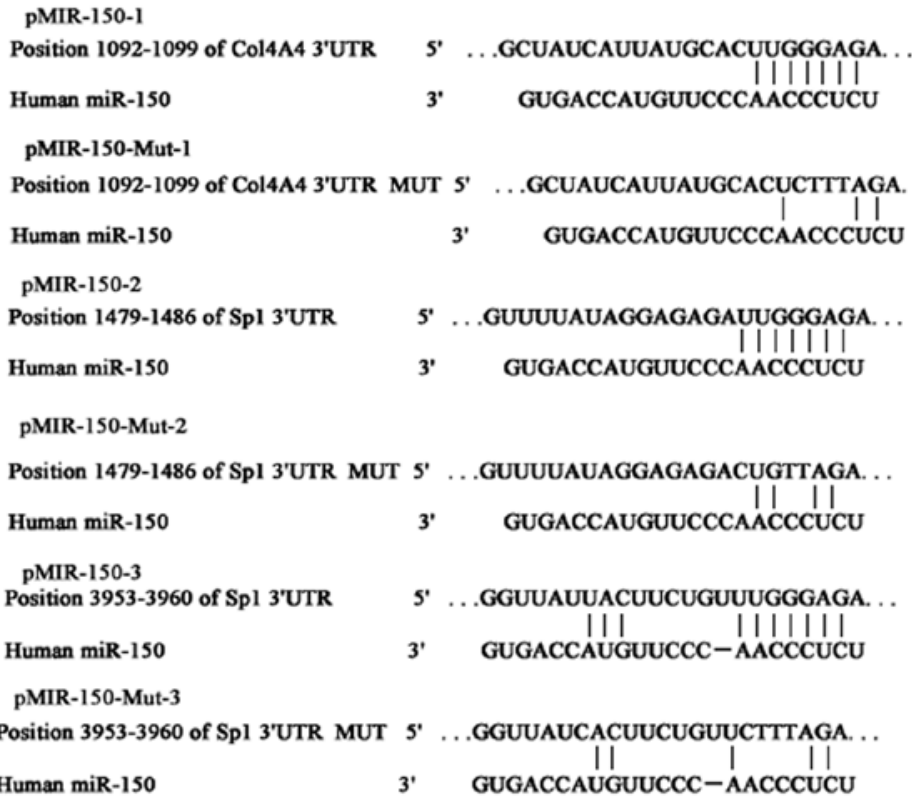

B

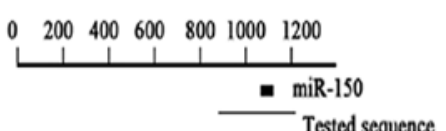

0100020003000400050006000

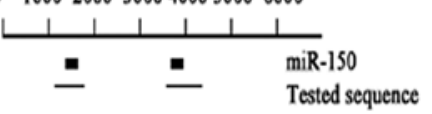

D
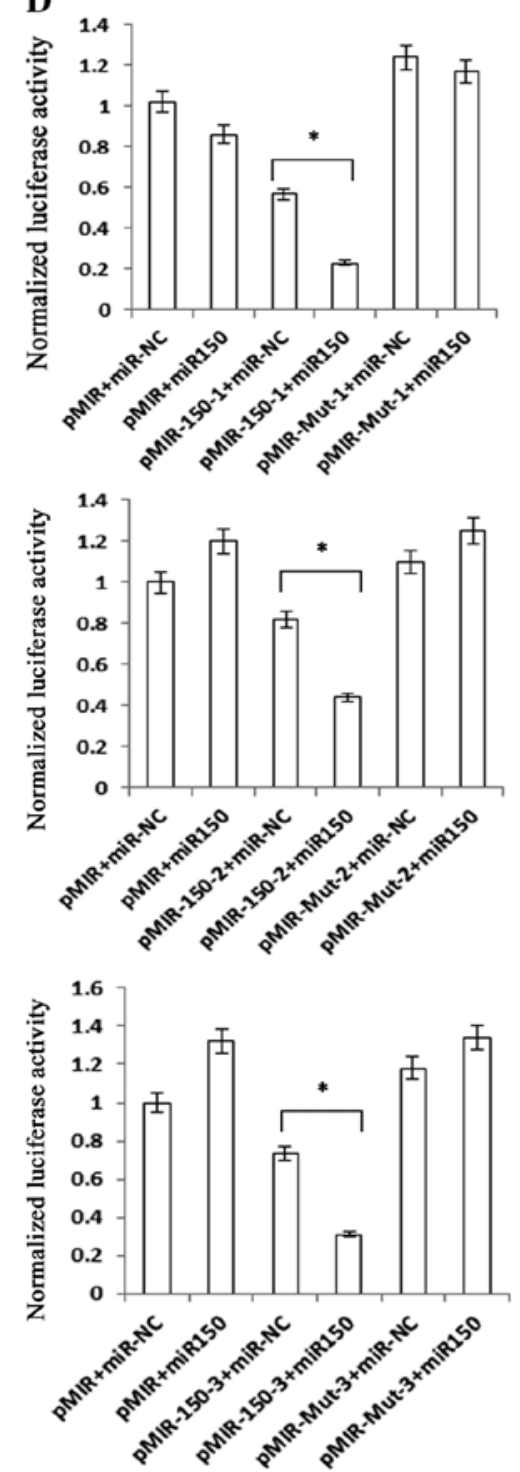

Figure 4. Interaction of miR-150 with the 3'UTRs of Col4A4 and Sp1. (A) The mRNA expression levels of Col4A4 and Sp1 were analyzed by real-time PCR. ${ }^{\prime} \mathrm{P}<0.05$. (B) Schematic indication of the miRNA binding sites in the 3'UTRs of Col4A4 and Sp1 mRNAs based on TargetScan Human Release 6.2. Black boxes indicate miR-150 and tested sequences indicate the regions that were inserted into the luciferase reporter vector. (C) Predicted consequential pairing of the target region and miR-150. According to their pairing sites, the corresponding luciferase reporter vectors were named pMIR-150-1, pMIR-150-2, pMIR-150-3, pMIR-150-Mut-1, pMIR-150-Mut-2 and pMIR-150-Mut-3. (D) The human stellate cells were transfected with pMIR (empty vector), pMIR containing miR150 targeting sequence (pMIR-miR150) and pMIR with miR-150 mutated target sequence (pMIR-mut). The top graph shows luciferase activity after LX-2 cells transfected with pMIR-150-1 or pMIR-150-Mut-1. The middle graph shows luciferase activity after LX-2 cells transfected with pMIR-150-2 or pMIR150-Mut-2. The bottom graph shows luciferase activity after LX-2 cells transfected with pMIR-150-3 or pMIR-150-Mut-3. All graphs also show cotransfection of miR-150 precursor or miR-NC. ${ }^{*} \mathrm{P}<0.05$.

$72 \mathrm{~h}$ after the treatment of TGF- $\beta 1$. These findings raised the possibility that miR-150 may play a pivotal role in the progression of liver fibrosis.

Effects of miR-150 overexpression on the activation of $L X-2$ cells. Next, we investigated the effects of miR-150 overexpression on the activation of HSCs. When compared with the control, the mRNA expression levels of CollA1 and Col1A2 in cells transfected with miR-150 mimics were markedly suppressed to 27.1 and $52.3 \%$, respectively (Fig. 3A). The miR-150 mimics also significantly reduced the mRNA expression level of $\alpha$-SMA to $31.1 \%$. It was further confirmed that all the mRNA expression levels of Col1A1, Col1A2 and $\alpha$-SMA were inhibited in miR-150-overexpressing cells under the stimulation of TGF- $\beta 1$. Consistent with the results of the mRNA expression levels, immunoblot analysis showed that the protein expression levels of type I collagen and $\alpha$-SMA were also suppressed by the overexpression of miR-150 (Fig. 3B). Then, we examined the role of miR-150 in regulating the proliferation and apoptosis of LX-2 cells. As shown by the MTT assay, the cells transfected with miR-150 mimics had significantly slower growth rates than the control, which was inhibited to $62.1 \%$ (Fig. 3D). However, there was no significant change in apoptosis rate in the cells transfected with miR-150 mimics when compared with the control (Fig. 3E). These data suggested that the expression of miR-150 reduced the production of ECM protein and suppressed HSC activation, whereas it did not influence the survival of LX-2 cells. 

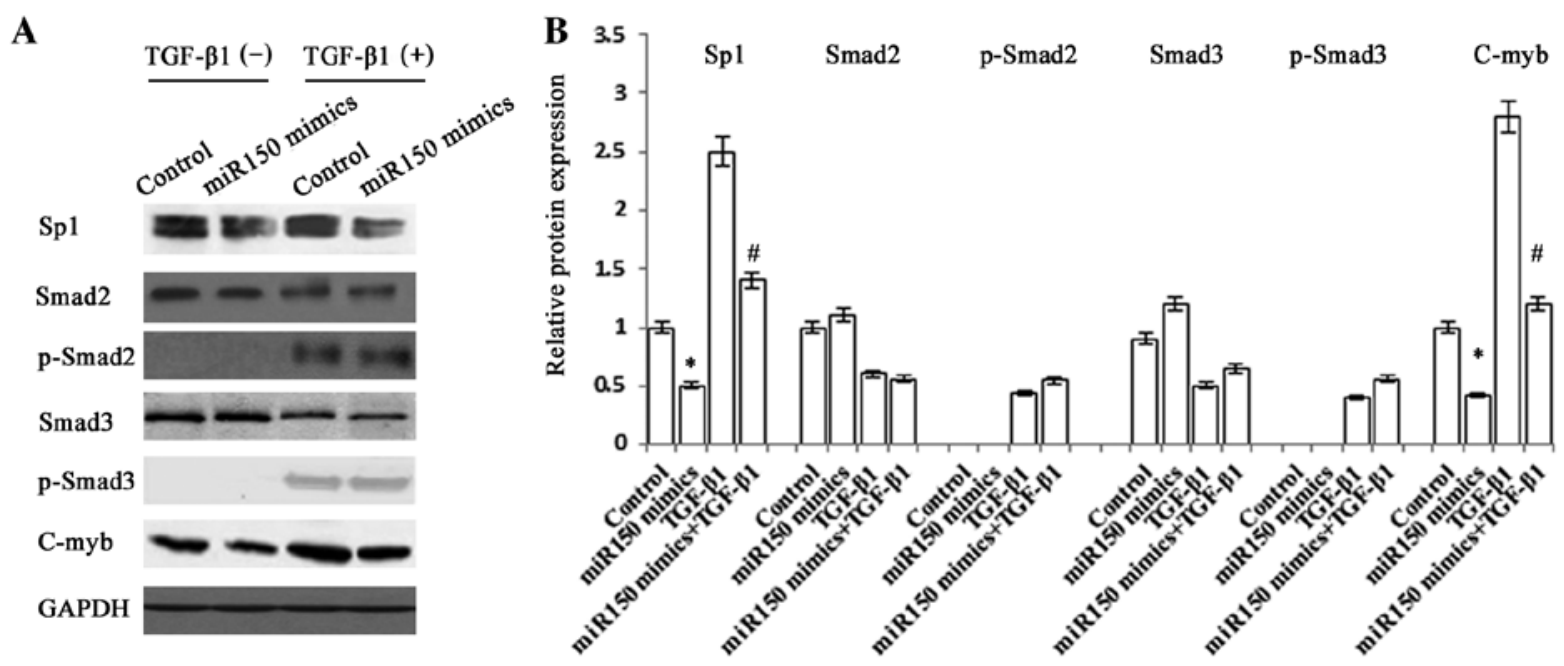

Figure 5. Regulation of type I collagen expression by miR-150 in LX-2 cells. (A) Effect of miR-150 overexpression on the protein expressions of Sp1, phosphoSmad2 (p-Smad2), Smad2, p-Smad3, Smad3, C-myb and GAPDH in LX-2 cells in the presence (+) or absence (-) of TGF- $\beta 1$. (B) Statistical analysis of Smad2, p-Smad2, Smad3, p-Smad3, Sp1 and C-myb protein expressions. Bars represent mean and standard error of three independent experiments. "P<0.05 compared with the control. ${ }^{*} \mathrm{P}<0.05$ compared with the TGF- $\beta 1$ group.

Col4A4 and Sp1 are the targets of miR-150. To explore the molecular mechanism of miR-150 in the collagen production of HSCs, we predicted the targets of miR-150 using bioinformatics analysis. We predicted that Col1A1 and Col1A2 were not targets of miR-150. Therefore, we conjectured that the reduction of Col1A1 and Col1A2 may be controlled by other mechanisms. It has been demonstrated that the expression of Col1A1 mRNA induced by TGF- $\beta$ is through a pathway including Sp1 and phosphorylated Smad2/3 (21). The promoter activity of CollA1 could be reduced by the knockdown of $\mathrm{Sp} 1$ and Smad2. Since Sp1 is a transcriptional regulator of Col1A1 expression induced by TGF- $\beta$, we considered whether it was a potential target gene for miR-150. We predicted that miR-150 could interact with the 3'UTRs of human Col4A4 and Sp1 mRNA using TargetScan Human Release 6.2 (http://www. targetscan.org/) (Fig. 4B and C). The sequence of each target region including the 3'UTRs of Col4A4 and Sp1 mRNA was cloned into pMIR-Report ${ }^{\mathrm{TM}}$ Luciferase plasmid. The construct was cotransfected into LX-2 cells along with miR-150 precursor or miR-NC. $\beta$-gal reporter control plasmid was cotransfected to monitor transfection efficiency. miR-150 precursor significantly reduced luciferase activities driven by the wild-type 3'UTRs of Col4A4 and Sp1 when compared with their respective miR-NC. By contrast, miR-150 precursor could not inhibit luciferase activities of mutated type Col4A4 3'UTR, mutated type Sp1 3'UTR and empty vector (Fig. 4D). To further verify the above results, we measured the mRNA and protein expression levels of Col4A4 and Sp1 in cells transfected with miR-150 mimics. As expected, the overexpression of miR-150 not only decreased the mRNA expressions of Col4A4 and Sp1, but also reduced the protein expressions of type IV collagen and Sp1 (Figs. 3B, 4A and 5A). These results suggested that Col4A4 and Sp1 are the targets of miR-150.

Regulation of type I collagen expression by miR-150. The degradation of type IV collagen could be directly induced by miR-150, but the underlying molecular mechanism of the reduction of type I collagen and $\alpha$-SMA remains unclear.
Next, further study was performed to confirm whether the pathway including Sp1 and Smad2/3 is responsible for the reduction of Col1A1. There was a significant decrease in the protein expression levels of $\mathrm{Sp} 1$ in miR-150-overexpressing cells (Fig.5). However, these cells showed no significant change in the protein expression levels of Smad2, p-Smad2, Smad3 and p-Smad3. These data showed that the upstream of TGF- $\beta$ signaling pathway was not affected by the effect of miR-150. We also found that C-myb, which is a target of miR-150 and plays a role in regulating the expression of $\alpha$-SMA and ECM proteins such as type I collagen, was reduced in miR-150-overexpressing cells (15). These results suggested that miR-150 reduces the production of type I collagen via a complex pathway including the regulation of C-myb and Sp1.

\section{Discussion}

HSCs play an important role in the progression of hepatic fibrosis and are activated by liver inflammation factors or injuries $(22,23)$. The activated HSCs undergo proliferation and secrete excessive ECM proteins such as type I and IV collagen (24). The type I collagen, which is encoded by Col1A1 and Col1A2, accounts for $36 \%$ of the total collagens in ECM of healthy liver and deposits in the perisinusoidal space during liver fibrogenesis (25). The most markedly upregulated collagen is the type IV collagen in liver fibrosis, which constitutes less than $10 \%$ of total collagen in the normal liver (26-28). The objective of the present study was to explore the antifibrotic effect of miR-150 as well as the underlying molecular mechanism of suppression of HSC activation by miR-150.

It is may be that different HSC behaviors are controlled by different miRNAs, particularly in the synthesis of the ECM protein. For example, miR-29b, which is a regulator of type I collagen mRNA and protein, could suppress the activation of HSCs $(29,30)$. In this study, the expression of miR-150 was found to be significantly reduced in the TGF- $\beta 1$ group in a dose- and time-dependent manner. Therefore, we speculated 
that miR-150, which is inhibited in LX-2 cells treated with TGF- $\beta 1$, may play a role in the antifibrotic effect. As expected, following the restoration of miR-150 in LX-2 cells, the mRNA and protein expressions of type I collagen and $\alpha$-SMA were markedly decreased and the cell growth rate was significantly inhibited, which is consistent with a previous study (15). Our data suggested that miR-150 could inhibit the production of ECM proteins and the activation of HSCs. However, there was no change in the apoptotic rate of DNA fragment in cells transfected with miR-150 as compared with the control, indicating that miR-150 did not affect the cell survival.

LX-2 cells, which are classified as an activated phenotype that expresses high levels of $\alpha$-SMA and collagens, are selected for all the experiments (31). Using TargetScan Human Release 6.2, we predicted that miR-150 may interact with the 3'UTR of Col4A4. It was further verified by the dual luciferase reporter experiment. Therefore, our data suggested that miR-150 was a potential regulator of type IV collagen mRNA and protein expressions. Furthermore, Sp1, which was a predicted target gene for miR-150, had two target regions for miR-150. Each of the target regions was cloned and inserted into the pMIR-Report ${ }^{\mathrm{TM}}$ luciferase plasmid. Our data demonstrated that miR-150 targets both target regions of 3'UTR of Sp1, which may be partly responsible for the reduction of Col1A1 mRNA and protein expressions in LX-2 cells transfected with miR-150 mimics. However, the Smad2 and phosphorylation of Smad2 expressions under TGF- $\beta 1$ stimulation, which are the downstream of the TGF- $\beta 1$ pathway, showed no change in LX-2 cells overexpressing miR-150 as compared with the control. The same was also seen in the expression of the Smad3 and phosphorylation of Smad3. Therefore, it is considered that miR-150 may affect the downstream of $\mathrm{Smad} 2 / 3$. In our study, it is not evident how miR-150 regulates the expression of Col1A2 and $\alpha$-SMA. We hypothesize that the reduction of Col1A2 and $\alpha$-SMA may be associated with $\mathrm{C}$-myb, which has been proved to be a target of miR-150 and to play a role in promoting the activation of HSCs (15). It has been found that C-myb expression is increased in activated HSCs and is associated with the development of fibrosis in animal models (32). Moreover, Lee et al (33) found that the type I collagen and $\alpha$-SMA could be induced via C-myb under the stimulation of oxidative stress. Our study confirmed that the overexpression of miR-150 reduced the $\mathrm{C}-\mathrm{myb}$ protein expression.

Collectively, our study illustrates that miR-150, which is crucial in the development of liver fibrosis, could inhibit type I and IV collagen in activated HSCs, at least in part, via the reduction of $\mathrm{Sp} 1$ and Col4A4 expression.

\section{Acknowledgements}

The study was supported by the Zhejiang Extremely Key Subject of Surgery, National Natural Science Foundation of China (81000176/H0317 and 81100292/H0317), the Zhejiang Provincial Natural Science Foundation of China (Y2090326 and Y2110634), the Wang Bao-En Liver Fibrosis Foundation (no. 20100002 and 20120127), the Wenzhou Municipal Science and Technology Bureau (Y20110033 and Y20120127) and the key disciplines in Colleges and Universities of Zhejiang Province.

\section{References}

1. Schuppan D, Krebs A, Bauer M and Hahn EG: Hepatitis C and liver fibrosis. Cell Death Differ 10 (Suppl 1): S59-S67, 2003.

2. Gressner OA, Rizk MS, Kovalenko E, Weiskirchen R and Gressner AM: Changing the pathogenetic roadmap of liver fibrosis? Where did it start; where will it go? J Gastroenterol Hepatol 23: 1024-1035, 2008.

3. Kong X, Horiguchi N, Mori M and Gao B: Cytokines and STATs in liver fibrosis. Front Physiol 3: 69, 2012.

4. Friedman SL: Mechanisms of hepatic fibrogenesis. Gastroenterology 134: 1655-1669, 2008.

5. Vettori S, Gay S and Distler O: Role of microRNAs in fibrosis. Open Rheumatol J 6: 130-139, 2012.

6. Berezikov E, Guryev V, van de Belt J, Wienholds E, Plasterk RH and Cuppen E: Phylogenetic shadowing and computational identification of human microRNA genes. Cell 120: 21-24, 2005.

7. Kozomara A and Griffiths-Jones S: miRBase: integrating microRNA annotation and deep-sequencing data. Nucleic Acids Res 39: D152-D157, 2011.

8. Brennecke J, Hipfner DR, Stark A, Russell RB and Cohen SM bantam encodes a developmentally regulated microRNA that controls cell proliferation and regulates the proapoptotic gene hid in Drosophila. Cell 113: 25-36, 2003.

9. Schratt GM, Tuebing F, Nigh EA, et al: A brain-specific microRNA regulates dendritic spine development. Nature 439: 283-289, 2006.

10. Chen CZ, Li L, Lodish HF and Bartel DP: MicroRNAs modulate hematopoietic lineage differentiation. Science 303: 83-86, 2004.

11. Mott JL, Kobayashi S, Bronk SF and Gores GJ: mir-29 regulates Mcl-1 protein expression and apoptosis. Oncogene 26: 6133-6140, 2007.

12. Kloosterman WP and Plasterk RH: The diverse functions of microRNAs in animal development and disease. Dev Cell 11: 441-450, 2006.

13. Chen L, Jiang M, Yuan W and Tang H: miR-17-5p as a novel prognostic marker for hepatocellular carcinoma. J Invest Surg 25: 156-161, 2012.

14. Yang F, Yin Y, Wang F, et al: miR-17-5p promotes migration of human hepatocellular carcinoma cells through the p38 mitogen-activated protein kinase-heat shock protein 27 pathway. Hepatology 51: 1614-1623, 2010.

15. Venugopal SK, Jiang J, Kim TH, et al: Liver fibrosis causes downregulation of miRNA-150 and miRNA-194 in hepatic stellate cells, and their overexpression causes decreased stellate cell activation. Am J Physiol Gastrointest Liver Physiol 298: G101-G106, 2010.

16. Ji J, Zhang J, Huang G, Qian J, Wang X and Mei S: Overexpressed microRNA-27a and $27 \mathrm{~b}$ influence fat accumulation and cell proliferation during rat hepatic stellate cell activation. FEBS Lett 583: 759-766, 2009.

17. Iizuka M, Ogawa T, Enomoto M, et al: Induction of microRNA$214-5 p$ in human and rodent liver fibrosis. Fibrogenesis Tissue Repair 5: 12, 2012.

18. Schmittgen TD and Livak KJ: Analyzing real-time PCR data by the comparative C(T) method. Nat Protoc 3: 1101-1108, 2008.

19. Thompson WJ, Piazza GA, Li H, et al: Exisulind induction of apoptosis involves guanosine 3',5'-cyclic monophosphate phosphodiesterase inhibition, protein kinase $\mathrm{G}$ activation, and attenuated beta-catenin. Cancer Res 60: 3338-3342, 2000.

20. Gressner AM, Weiskirchen R, Breitkopf K and Dooley S: Roles of TGF-beta in hepatic fibrosis. Front Biosci 7: d793-d807, 2002.

21. Sysa P, Potter JJ, Liu X and Mezey E: Transforming growth factor-beta1 up-regulation of human alpha(1)(I) collagen is mediated by Sp1 and Smad2 transacting factors. DNA Cell Biol 28: 425-434, 2009.

22. Bataller R and Brenner DA: Hepatic stellate cells as a target for the treatment of liver fibrosis. Semin Liver Dis 21: 437-451, 2001.

23. Friedman SL: Molecular regulation of hepatic fibrosis, an integrated cellular response to tissue injury. J Biol Chem 275: 2247-2250, 2000

24. Wu J and Zern MA: Hepatic stellate cells: a target for the treatment of liver fibrosis. J Gastroenterol 35: 665-672, 2000

25. Kwiecinski M, Noetel A, Elfimova N, et al: Hepatocyte growth factor (HGF) inhibits collagen I and IV synthesis in hepatic stellate cells by miRNA-29 induction. PLoS One 6: e24568, 2011. 
26. Gressner AM and Weiskirchen R: Modern pathogenetic concepts of liver fibrosis suggest stellate cells and TGF-beta as major players and therapeutic targets. J Cell Mol Med 10: 76-99, 2006.

27. Geerts A, Schuppan D, Lazeroms S, De Zanger R and Wisse E: Collagen type I and III occur together in hybrid fibrils in the space of Disse of normal rat liver. Hepatology 12: 233-241, 1990.

28. Milani S, Herbst H, Schuppan D, Surrenti C, Riecken EO and Stein H: Cellular localization of type I III and IV procollagen gene transcripts in normal and fibrotic human liver. Am J Pathol 137: 59-70, 1990.

29. Sekiya Y, Ogawa T, Yoshizato K, Ikeda K and Kawada N: Suppression of hepatic stellate cell activation by microRNA-29b. Biochem Biophys Res Commun 412: 74-79, 2011.
30. Ogawa T, Iizuka M, Sekiya Y, Yoshizato K, Ikeda K and Kawada N: Suppression of type I collagen production by microRNA-29b in cultured human stellate cells. Biochem Biophys Res Commun 391: 316-321, 2010.

31. Xu L, Hui AY, Albanis E, et al: Human hepatic stellate cell lines, LX-1 and LX-2: new tools for analysis of hepatic fibrosis. Gut 54: $142-151,2005$.

32. Kitada T, Seki S, Nakatani K, Kawada N, Kuroki T and Monna T: Hepatic expression of c-Myb in chronic human liver disease. Hepatology 26: 1506-1512, 1997.

33. Lee KS, Buck M, Houglum K and Chojkier M: Activation of hepatic stellate cells by TGF alpha and collagen type I is mediated by oxidative stress through c-myb expression. J Clin Invest 96: 2461-2468, 1995. 